\title{
Superpixel-based Prostate Cancer Detection from Diffusion Magnetic Resonance Imaging
}

Junjie Zhang

Farzad Khalvati

Alexander Wong

Masoom A. Haider
University of Toronto, ON, Canada

University of Toronto, ON, Canada

University of Waterloo, ON, Canada

University of Toronto, ON, Canada

\section{Abstract}

This paper presents a superpixel-based approach to detect prostate cancers from diffusion magnetic resonance imaging (dMRI). In this approach, superpixel generated candidate regions are incorporated in the quantitative radiomics model, MAPS [1], to detect prostate cancers from dMRI modalities. Experimental results show the feasibility of the proposed superpixel-based approach with improved computation efficiency and detection accuracy.

\section{Introduction}

Automatic prostate cancer detection from MRI has been an area of intense research due to the increased use of MRI as modality for the clinical diagnosis of prostate cancer. In particular, recent researches have focused on diffusion MRI, which quantifies the diffusion of water molecules in tissue, as it has shown promise in differentiating healthy and tumorous tissue [1]. Conventional MRI prostate cancer auto-detection consists of deriving low-level features from one or more MRI modalities and applying a classifier to the derived features. Those methods are primarily based on pixels, which can be computationally inefficient and not robust to the intrinsic noisy MRI data.

Motivated to improve the efficiency and robustness of computer-aided prostate cancer detection from MRI, this paper proposes a superpixel-based approach to address these drawbacks in conventional methods. The presented method treats superpixels as the basic units for higherlevel object representation, which allows for reduced computational cost, while potentially increasing the detection accuracy, as it is more robust to noise than pixels-based method.

\section{Methodology}

The proposed approach can be summarized in three steps: (1) Superpixel segmentation of prostate gland; (2) MAPS feature extraction; and (3) Classification and validation.

In the first step, an improved variation of the superpixel segmentation algorithm SLIC [2] is applied to segment prostate gland in ADC to generate candidate regions. This algorithm is selected for its reported performances of well adherence to image boundaries and high computational efficiency. The behaviour of the clustering algorithm resembles $k$-means, but limits the searching space to a region proportional to the superpixel size. While the distance measure in [2] is defined in a 5-D space ( $x, y$ position and r.g.b converted $L, a, b$ values in CIELAB color space), in our study, the $L, a, b$ distance is replaced with intensity difference in MRI (ADC) images. So the distance function $D$ is written as:

$$
\begin{aligned}
& d_{I}=\left|I_{j}-I_{i}\right| \\
& d_{s}=\sqrt{\left(x_{j}-x_{i}\right)^{2}+\left(y_{j}-y_{i}\right)^{2}} \\
& D=\sqrt{\left(\frac{d_{I}}{m_{I}}\right)^{2}+\left(\frac{d_{s}}{m_{s}}\right)^{2}}
\end{aligned}
$$

where $m_{I}$ and $m_{s}$ are the maximum intensity and spatial distances of each cluster observed from previous iteration.
Fig. 1 illustrates the superpixel segmentation of a prostate gland in ADC.

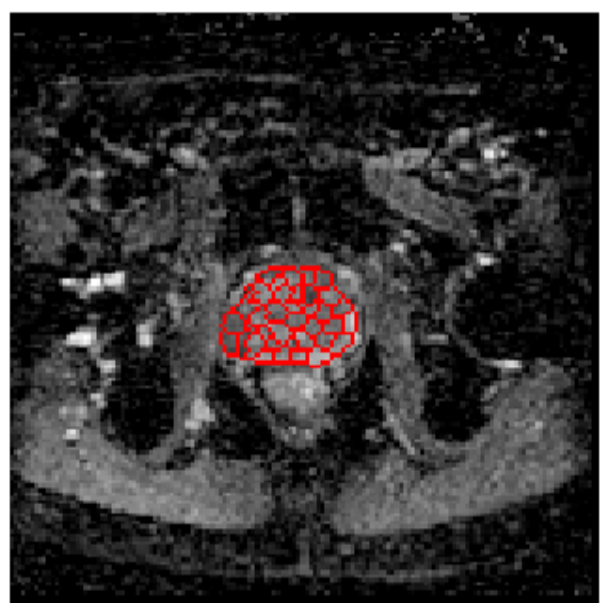

Fig. 1: Superpixel segmentation of prostate gland

In steps 2 and 3, the generated superpixels are then incorporated into the quantitative radiomics model, MAPS, developed in [1], to detect prostate cancer.

\section{Results and Discussion}

The proposed approach was tested using actual clinical MRI data imaged from eleven patients. The preliminary results obtained were promising: region-based statistics showing an accuracy of $98.7 \%$ and specificity of $98.9 \%$, though with a sensitivity of $81.8 \%$.

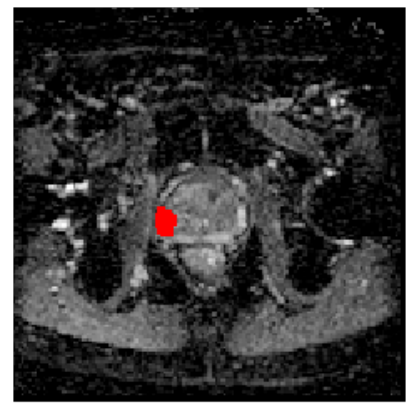

(a)

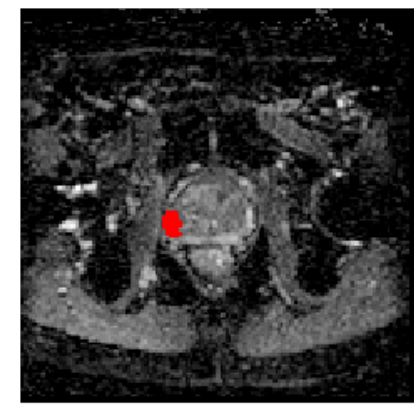

(b)
Fig. 2: Comparison between prostate cancer region delineated by the radiologist (a) and that obtained by superpixel-based approach (b)

Fig. 2 shows a comparison between prostate cancer region delineated by an experienced radiologist and that obtained by the proposed superpixel-based approach. The results demonstrate the potential of this approach for computeraided prostate cancer screening and diagnosis.

\section{References}

[1] Cameron, A., Khalvati, F., Haider, M. and Wong, A. MAPS: A Quantitative Radiomics Approach for Prostate Cancer Detection. IEEE Transactions on Biomedical Engineering (2015).

[2] Achanta, R., Shaji, A., Lucchi, A., Fua, P. and Susstrunk S. SLIC Superpixels Compared to State-ofthe-Art Superpixel Methods. IEEE Trans. Pattern Analysis and Machine Intelligence (2012). 